\title{
Noninflammatory Diffuse Follicular Hypertrophy/ Hyperplasia of Graves Disease: Morphometric Evaluation in an Experimental Mouse Model
}

\author{
Anke Schlüter ${ }^{a, b}$ Anja K. Eckstein ${ }^{a, c}$ Alexandra Brenzeld Mareike Horstmann ${ }^{a}$ \\ Stephan Lang ${ }^{b}$ Utta Berchner-Pfannschmidt ${ }^{a} \quad$ J.Paul Banga ${ }^{a}$ \\ Salvador Diaz-Cano ${ }^{\mathrm{e}}$ \\ a Molecular Ophthalmology, Department of Ophthalmology, University Hospital Essen, Essen, Germany; \\ ${ }^{b}$ Department of Oto-Rhino-Laryngology - Head and Neck Surgery, University Hospital Essen, Essen, Germany; \\ 'Department of Ophthalmology, University Hospital Essen, Essen, Germany; ${ }^{\mathrm{d}}$ Imaging Center Essen (IMCES), \\ Institute for Experimental Immunology and Imaging, University Hospital Essen, Essen, Germany; ${ }^{\mathrm{e} D e p a r t m e n t}$ of \\ Histopathology, King's College Hospital NHS, London, UK
}

\section{Keywords}

Graves disease $\cdot$ Hypertrophy/hyperplasia $\cdot$ Glandular involvement · Follicular expansion · Morphometric evaluation

\begin{abstract}
Objectives: Experimental models of Graves hyperthyroid disease accompanied by Graves orbitopathy (GO) can be efficiently induced in susceptible inbred strains of mice by immunization by electroporation of heterologous human TSH receptor (TSHR) A-subunit plasmid. The interrelated pathological findings in the thyroid glands of Graves disease (GD) that explain the core changes classically include diffuse follicular hyperplasia and multifocal mild lymphocytic infiltrate. However, the relative contributions of different thyroid tissue components (colloid, follicular cells, and stroma) have not been previously evaluated. In this study, we characterize the thyroid gland of an experimental mouse model of autoimmune GD. Our objective was to define the relative contribution of the different thyroid tissue components to the pathology of glands in the experimental model. Methods: Mice were immunized with human TSHR A-subunit plasmid. Anti-
\end{abstract}

\section{KARGER}

(c) 2018 European Thyroid Association

Published by S. Karger AG, Basel

E-Mail karger@karger.com

www.karger.com/etj bodies induced to human TSHR were pathogenic in vivo due to their cross-reactivity to mouse TSHR. Results: Autoimmune thyroid disease in the model was characterized by histopathology of hyperplastic glands with large follicular cells. Further examination of thyroid glands of immunized animals revealed a significantly increased follicular area and follicle/stroma ratio, morphometrically correlated with a noninflammatory follicular hyperplasia/hypertrophy. The increased follicle/stroma ratio was the most relevant morphometrically variable summarizing the pathological changes for screening purposes. Conclusion: GD thyroid glands are enlarged and characterized by a noninflammatory diffuse follicular cell hyperplasia/hypertrophy and a significant increase in the follicles with an increased follicle/stroma ratio. Overall, this mouse model is a faithful model of an early hyperthyroid status of GD (diffuse glandular involvement and follicular expansion).

(C) 2018 European Thyroid Association Published by S. Karger AG, Basel

Dr. med. Anke Schlüter, MD

Department of Oto-Rhino-Laryngology - Head and Neck Surgery

University Hospital Essen, Hufelandstrasse 55

DE-45147 Essen (Germany)

E-Mail anke.schlueter@uk-essen.de

Dr. Salvador Diaz-Cano, MD, PhD, FRCPath

Department of Histopathology, King's College Hospital

Denmark Hill

London SE5 9RS (UK)

E-Mail sdiaz-cano@nhs.net 


\section{Introduction}

Graves disease (GD) is caused by autoantibodies that induce hyperthyroidism by mimicking the action of TSH and activating the TSH receptor (TSHR). The defining component of hyperthyroidism is thyroid follicular hyperplasia secondary to TSHR stimulation, that, together with additional findings (ophthalmopathy, dermopathy), constitutes the typical clinical triad of GD pathology [14]. Because of human diversity as well as distinct ethical limitations, syngeneic animal models of hyperplastic and autoimmune diseases are invaluable investigative tools for complex pathological conditions such as GD and autoimmune thyroiditis [5-7]. We have recently reported the development of a robust and reproducible model of experimental GD that also develops the complication of Graves orbitopathy (GO) in female BALB/c mice due to immunization by electroporation with plasmid encoding heterologous human TSHR ectodomain (human TSHR A-subunit) $[8,9]$, but did not describe the thyroid pathology in detail.

The thyroid conditions GD and Hashimoto thyroiditis (hypothyroidism) span two extremes of autoimmune disorders, and not surprisingly share many underlying tenets. Although such thyroid-stimulating autoantibodies are pathognomonic of GD, autoantibodies to thyroid peroxidase (TPO) and thyroglobulin (Tg) are also present [1, 10]. Moreover, some GD patients have mild lymphocytic thyroiditis. In some instances, thyroiditis in GD becomes sufficiently extensive as to cure the hyperthyroidism with resultant hypothyroidism. Thyroid-stimulating antibodies, the proximal cause of Graves hyperthyroidism, arise from the breakdown in self-tolerance to the TSHR [11]. The pathophysiological relationship between TSHR, $\mathrm{TPO}$, and Tg autoantibodies remains an enigma, and it is unknown whether TSHR or the other autoantigens are associated with the lymphocytic infiltration in GD [1215]. The crucial role of anti-TSHR antibodies in the pathogenesis of hyperthyroidism is supported by animal models of GD. The autoantigen that drives the immune response in GD is not the full-length TSHR, but the Asubunit, an ectodomain component that is shed after intramolecular cleavage of the receptor [16]. Genetic immunization of female inbred mice by electroporation with plasmid encoding human TSHR A-subunit cDNA, or indeed autologous mouse TSHR A-subunit, results in the generation of pathogenic cross-reactive antibodies that cause thyroid stimulation, thyroid enlargement with lymphocytic infiltration, elevated thyroxine levels, and in a subset of mice, ocular signs reminiscent of GO [12, 13,
17]. Additionally, the GD histopathological changes are heterogeneous and subtle in many cases, including a mixture of the initial hyperplastic reaction, along with the changes induced by the presurgical therapeutic intervention (e.g., Lugol) or antithyroid drug therapy.

In human GD, the thyroid gland pathology is difficult to determine in early stages of disease due to lack of availability of the gland. An understanding of thyroid pathology and contributing components in early disease stages will help to define relevant mechanisms through which the cascade of thyroid follicular hyperplasia sets in the condition. Moreover, the relative contribution of different thyroid tissue components (colloid, follicular cells, and stroma) in thyroid pathology has not been previously evaluated, and hence the mechanisms remain unknown. In this study, we used the experimental mouse model of GD to study the thyroid tissue components and their contribution to the pathology of thyroid glands in early-stage disease.

\section{Methods}

Induction of Experimental GD

A total number of 31 female $B A L B / c$ mice were bred and housed in a specific pathogen-free environment in animal care facilities at University Hospital Essen. Investigations involving animals have been approved by the institutional ethics animal committee of North Rhine Westphalia State Agency for Nature, Environment and Consumer Protection, Germany. At the age of 6-8 weeks, the mice were immunized with intramuscular injection and electroporation of $50 \mu \mathrm{g}(1 \mathrm{mg} / \mathrm{mL})$ plasmid into each biceps femoris muscle 4 times every 3 weeks $[8,9,17]$; 14 animals were immunized with pTRiEx1.1 human TSHR A-subunit plasmid and 17 animals with control B-Gal plasmid (Ctrl). Six weeks after the end of immunization, the mice were sacrificed, and serum was collected and stored at $-80^{\circ} \mathrm{C}$. In light of observing regulations for animal studies on 3Rs (replacement, reduction, refinement), the group of TSHR-immunized female animals $(n=14)$ was an experiment that was running concurrently with another group of immune animals for a different study. Hence, the results of this female group were also used in data analysis for a different study dealing with the influence of gender in a GD/GO model (submitted for publication).

\section{Serological Parameters}

Anti-TSHR antibodies were measured in serum as TSH binding inhibiting immunoglobulins using commercial TRAK kits (BRAHMS Thermo Fisher). The total $\mathrm{T}_{4}$ level was analyzed in the sera by ELISA following the suppliers' instructions (total $\mathrm{T}_{4}$ ELISA; DRG, Springfield, NJ, USA).

\section{Thyroid Pathology}

Microsurgical excised thyroid glands were fixed in buffered formalin and embedded in paraffin. Serial sections $(1 \mu \mathrm{m})$ from the middle region of the thyroid were stained using hematoxylin-eo- 
sin. Sections were scored blindly by an experienced observer as normal, hypoactive, and hyperactive state, referenced to the corresponding control group (equal to, below, and above matched animals). These histological categories try to set the baseline for the model in three groups of early stages of disease without any preconception regarding the findings.

\section{Cell Profiler Analyses}

Thyroid gland sections were analyzed using Cell Profiler (Broad Institute of Harvard, MIT). Images were generated using an Olympus BX51 microscope (Fig. 1). Cell Profiler allows a standardized interactive exploration and analysis of data. A pipeline was programmed to analyze thyroid tissue in the following way (measured tissue is shown in Table 1): first, the whole tissue was detected, and the blank area was excluded from the following measurements. The total area of the thyroid gland was measured $\left(\mathrm{mm}^{2}\right)$. Next, a nucleus mask was created by a threshold, and the number of nuclei was counted. For the following procedure, the nuclei were subtracted from the thyroid tissue, and connective tissue was suppressed by a suppression filter. Follicles (as well as nuclei) very close to each other or even attached were separated by shape and intensity water shedding method. Subsequently, structures of thyroid follicle were identified and quantified in area and number.

- Numbers of follicles were counted

- Area of colloid of the follicles was measured $\left(\mu \mathrm{m}^{2}\right)$

- Stromal tissue was measured $\left(\mu \mathrm{m}^{2}\right)$

Our morphometric approach identified the two main tissue components: follicular parenchymatous and stromal-vascular tissue. It also counted the number of follicular structures to identify the average follicular area. The number of follicles and the follicular area provide an accurate and straightforward morphological estimate of the functional gland.

\section{Statistical Analyses}

Data were statistically analyzed with GraphPad Prism Software (San Diego, CA, USA). The level of significance was set at $p<0.05$. Statistical analyses were carried out with two-tailed Student $t$ tests with a confidence level greater than $95 \%$. Data are presented as arithmetic mean \pm SEM. $p$ values are marked with stars representing $* p<0.05,{ }^{* *} p<0.01,{ }^{* * *} p<0.001$, and ${ }^{* * * *} p<0.0001$.

\section{Results}

\section{Serum Parameter}

Total $\mathrm{T}_{4}$ concentration in serum was significantly higher in TSHR-immunized mice than in control mice, and hence the animals were classified as hyperthyroid (7/14) $(p \leq 0.05)$ (online suppl. Fig. 1A; for all online suppl. material, see www.karger.com/doi/10.1159/000488079). Evaluation of serum for anti-TSHR antibodies showed all TSHR-immunized animals to be positive for TSH binding inhibitory immunoglobulins, while none of the control animals were positive (online suppl. Fig. 1B).

Follicular Hypertrophy/Hyperplasia of GD: Morphometric Evaluation
Table 1. An overview of the analyzed data

Analyzed tissue

Total area

Follicular area

Number of follicles

Number of nuclei $=$ number of cells

Stroma

The total area of the thyroid was measured, the follicular area was measured by the colloid area, numbers of follicles were counted, numbers of nuclei were counted to analyze how many cells were found in the thyroid, and the area of the stroma was measured.

\section{Thyroid Histology in GD Mice}

TSHR-immunized animals showed predominantly hyperplastic thyroids characterized by cuboid cylindrical follicular cells with a small amount of colloid (9/14 animals $=64 \%$ ). Only a few animals showed only one gland with hypothyroid features characterized by thin follicles $(1 / 14$ animals $=7 \%)$. Overall, 3 out of 9 hyperplastic animals (21\% of all TSHR-immunized mice) showed minor changes which could indicate different disease degrees, whereas 6 out of 9 hyperplastic thyroids showed significant changes (43\%). The remaining thyroid glands $(n=4,29 \%)$ from immunized animals showed no histological abnormalities, similar to control $ß$-galactosidase ( $\beta$-Gal)-immunized animals that were all scored as normal. The vascular component of the stroma was not significantly expanded in the routine histological evaluation.

\section{Total Area of Thyroid Glands}

Thyroid glands were analyzed objectively with Cell Profiler as described in Methods. The total area of thyroid glands was measured for each case in the middle area of serially sectioned organs (Fig. 1). For control B-Gal mice, a mean area of $1.2 \mathrm{~mm}^{2}$ was measured, while TSHR-immunized mice had an increased mean size of $1.9 \mathrm{~mm}^{2}$ $(p \leq 0.0001)$ (Fig. 2a).

\section{Follicular Cells}

The total number of follicle cell nuclei was comparable in both groups (Fig. 2b), while the number of nuclei per area $\left(\mathrm{mm}^{2}\right)$ was significantly reduced $(p \leq 0.01)$ in TSHRimmunized mice in comparison to control mice (Fig. 2c). This finding suggests that the average follicular cell area 


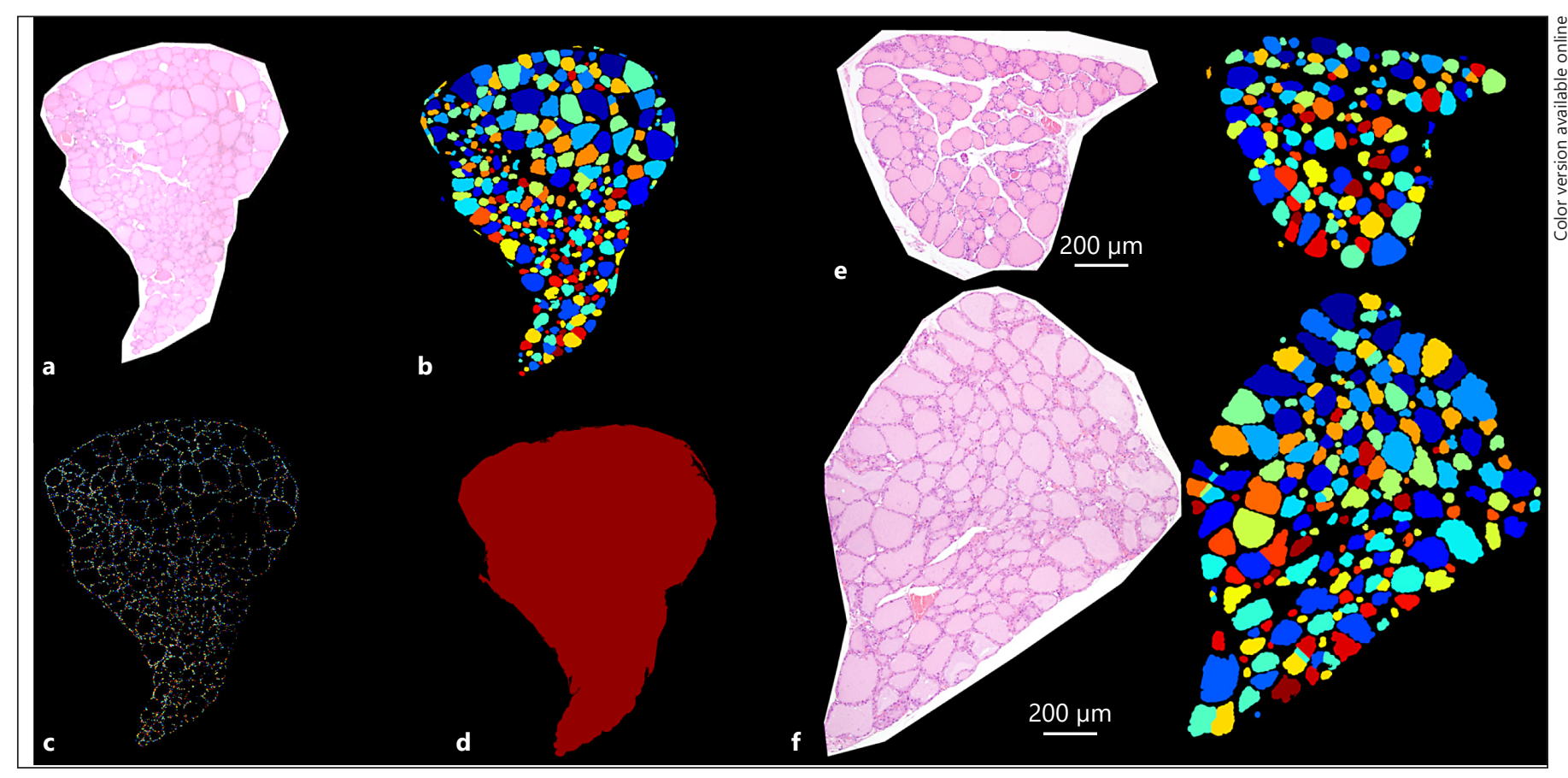

Fig. 1. Technical procedure of Cell Profiler and examples of both groups. Cell Profiler allows a standardized interactive exploration and analysis of data. a A Cell Profiler pipeline was programmed to analyze thyroid tissue in the following way: images were generated from each lobe using an Olympus BX51 microscope (100× magnification). Whole tissue was detected and the blank area, as well as fat tissue, was excluded from the following measurements. b Follicles (as well as nuclei) very close to each other or even attached were separated by the shape and intensity water shedding method. Subsequently, structures of thyroid follicle were identified and quantified in area and number. Numbers of follicles were counted. c The nuclei were subtracted from the thyroid tissue, and connective tissue was suppressed by a suppression filter. A nucleus mask was created by a threshold and counted the number of nuclei. d The total area of the thyroid was measured $\left(\mathrm{mm}^{2}\right)$. e, $\mathbf{f}$ Images with one thyroid lobe from $B-G a l$ control (e) and human TSHR A-subunit-immunized mice (f) with their particular water shedding methods. In this overview, it can be seen that the area of the thyroid in TSHR-immunized mice is more prominent than that in the $B-G a l$ control sample. Follicles are increased. Scale bars, 200 $\mu \mathrm{m}$. Magnification, $\times 100$. was increased in thyroid glands of the TSHR-immunized mice undergoing experimental GD.

\section{Follicular Area}

The total number of follicles was similar in both groups (Fig. 3a) while the number of follicles per area $\left(\mathrm{mm}^{2}\right)$ was more extensive in control mice (Fig. 3b). TSHR mice had significantly fewer follicles per area $(p \leq 0.0001)$, which suggests that the follicles were enlarged in TSHR-immunized mice, as seen in Figure 3c. Measuring the follicular area $\left(\mu \mathrm{m}^{2}\right)$ and determining the mean showed that TSHRimmunized mice had statistically significantly enlarged follicles ( $p \leq 0.0001)$. The mean follicular areas (MFA) of control mice was very similar to each other while that of TSHR-immunized mice had a significant dispersion, which supports the blind scoring of supposed different disease degrees. Analyzing MFA and the number of fol- licles in TSHR-immunized mice showed significantly higher MFA per follicle (Fig. 3d).

\section{Distribution of Follicles and Stroma, and Ratio}

To analyze the proportion of follicular tissue and stromal tissue the total area of follicles and the stromal area were measured (in $\mu \mathrm{m}^{2}$ ). Scores of the area of all follicles (Fig. 4a) and stroma (Fig. 4b) per total thyroid gland section were calculated. While the follicular area was increased in TSHR-immunized mice $(p \leq 0.01)$, the stromal tissue was dominant in control mice $(p \leq 0.01)$. The ratio of follicular area to stromal area was summarized in follicle/stroma scores (Fig. 4c) and was statistically significantly increased in TSHR mice in comparison to control mice $(p \leq 0.01)$. 


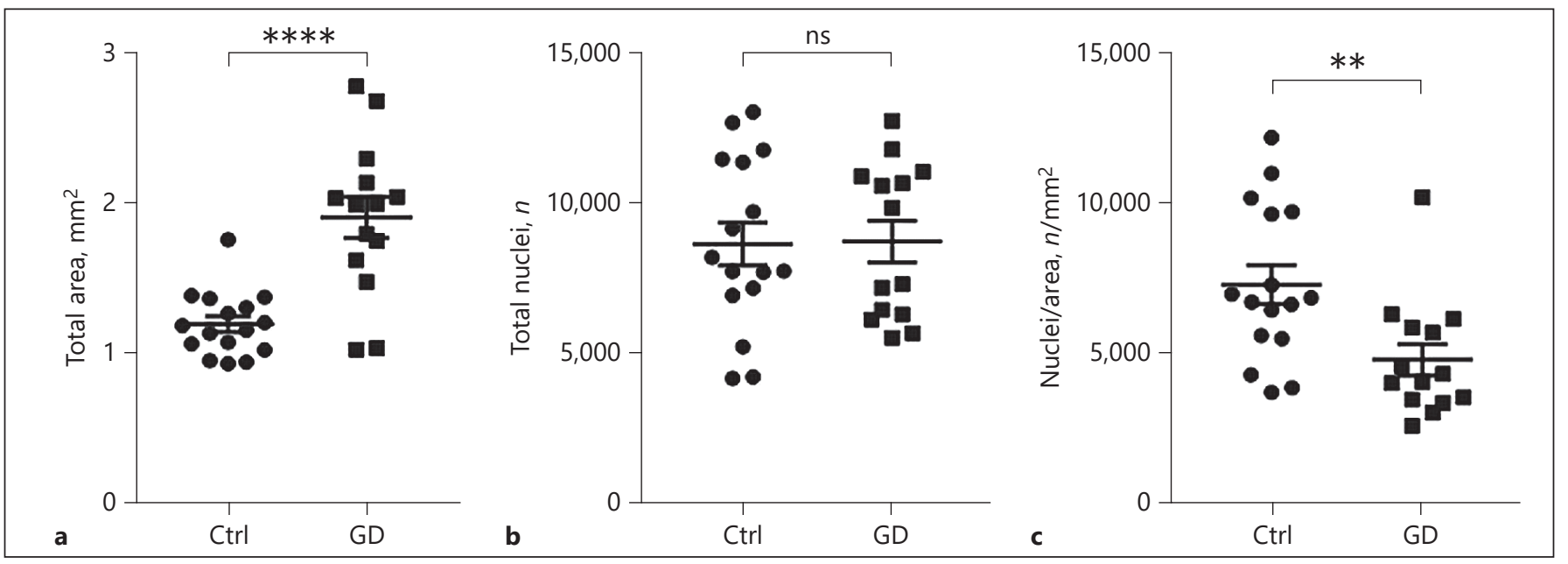

Fig. 2. Total area and number of cells. a Sections of $1 \mu \mathrm{m}$ were cut, and slices of the middle area of the thyroid were stained using hematoxylin-eosin. The total area of thyroid tissue was measured in $\mathrm{mm}^{2}$. TSHR-immunized mice (Graves disease, GD) showed a significantly increased area of thyroid in comparison to control $ß-\mathrm{Gal}$ mice (Ctrl). b The total number of nuclei was counted and showed no significant difference between the two groups. c The number of nuclei per area was measured and showed a higher number of nuclei in B-Gal control mice, which revealed that cells in TSHR-immunized mice seem to be more substantial because there are fewer nuclei in a larger area.
Fig. 3. Follicle analysis. a The total number of follicles was counted and showed no significant difference between the two groups (Graves disease, GD; control, Ctrl). b The number of follicles normalized to the total area was analyzed. TSHR-immunized mice showed statistically significantly fewer numbers of follicles per area, which led to the conclusion that the follicles were increased. ${ }^{* * *} p \leq 0.001$. $\mathrm{c}$ The mean follicular area was measured and analyzed. GD mice showed significantly increased follicle areas in comparison to $B-G a l$ control. $* * * * p \leq 0.001$. d Building a score of the mean follicular area (MFA) per number of follicles, GD mice showed a statistically significant higher score and enlarged follicular areas. ${ }^{* * * *} p \leq 0.001$.

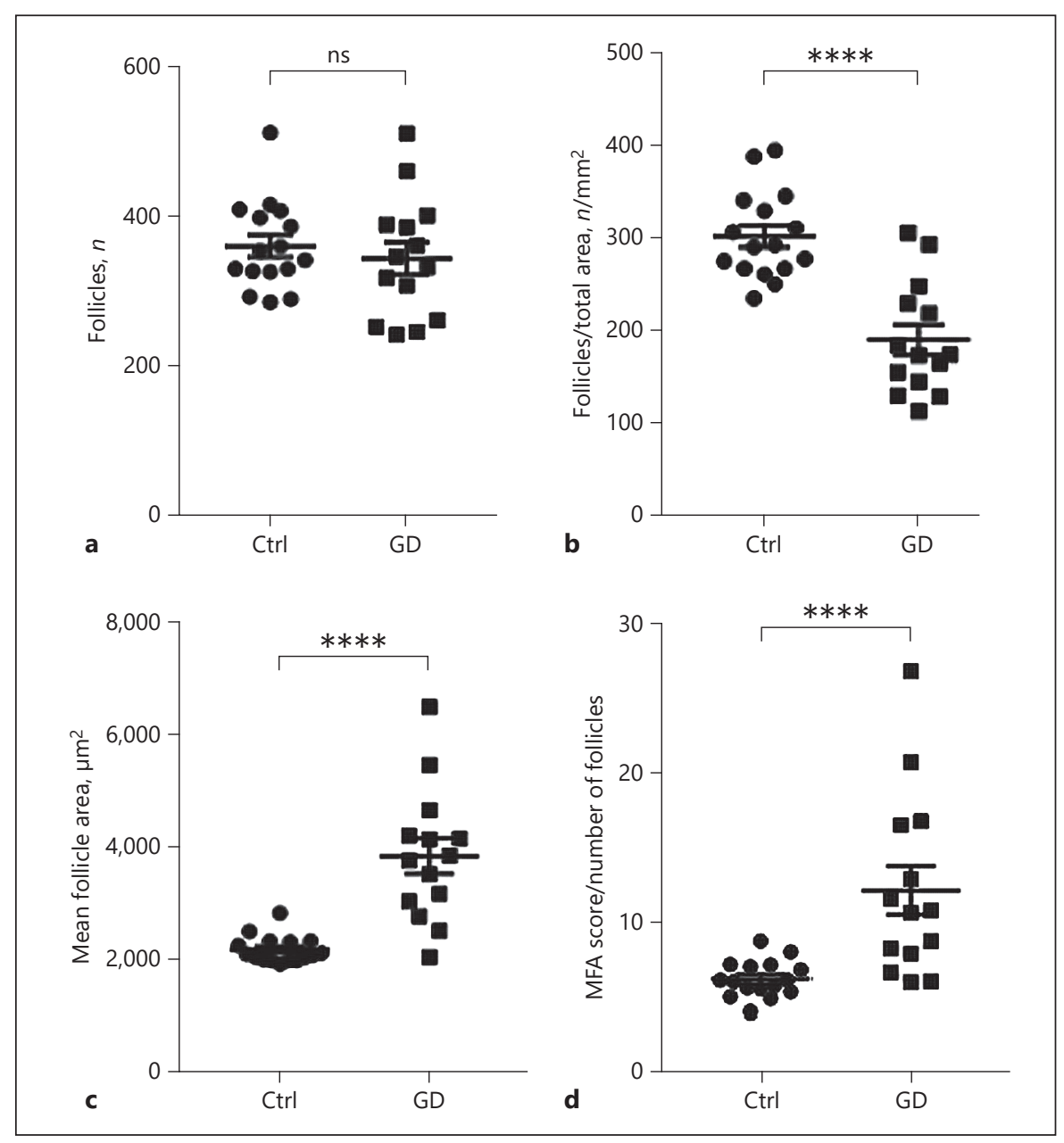

Follicular Hypertrophy/Hyperplasia of GD: Morphometric Evaluation
Eur Thyroid J

DOI: $10.1159 / 000488079$ 


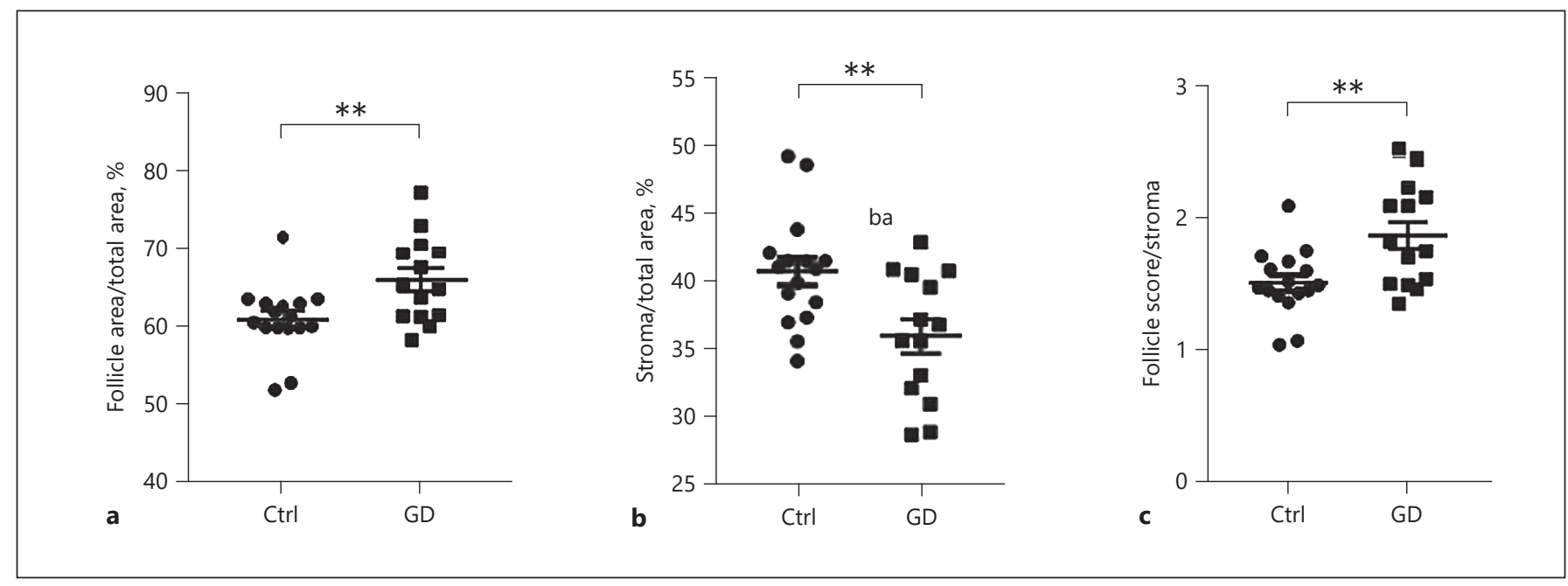

Fig. 4. Distribution of follicles and stroma. a The area of the follicle per total area was analyzed. Graves disease (GD) mice showed a significantly higher percentage of follicle area per total area in comparison to $\beta-G a l$ control mice (Ctrl). ${ }^{* *} p \leq 0.01$. b On the other hand, the percentage area of stroma was significantly more abundant in control mice than in GD mice. ${ }^{* *} p \leq 0.01$. c Building a score of follicles per stroma to analyze the relation of follicle area and stroma, GD mice showed a statistically significant higher ratio of follicle than stromal tissue. ${ }^{* *} p \leq 0.01$.

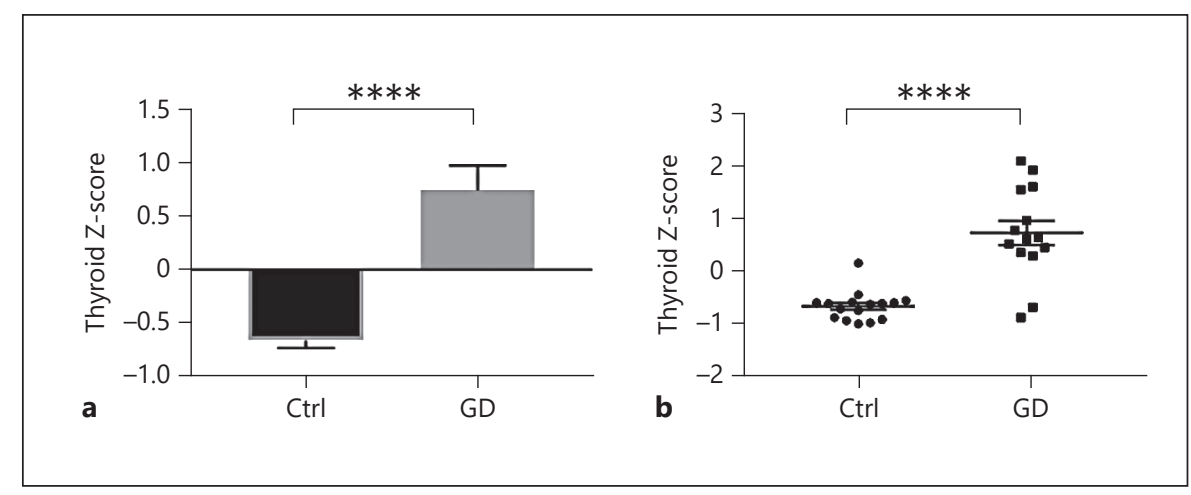

Fig. 5. Total disease Z-score. Results were summarized in a Z-Score to create a total thyroid disease score. The Z-score implicates the total area of thyroid, mean follicular area, and follicle/stroma score. a Overview of the results of the two groups (Graves disease, GD; control, Ctrl): the Z-score shows a statistically significant difference between the two groups. ${ }^{* * * *} p \leq 0.0001$. b Detailed view

\section{Total Disease Score}

To summarize the results, a Z-score was built to demonstrate the shaping of the total histological thyroid disease (Fig. 5). The Z-score implicates a total area of the thyroid, MFA, and follicle/stromal score. The Z-score showed a statistically significant $(p \leq 0.0001)$ difference between the two groups. All control mice showed a score $\leq 0$, as well as $14 \%(2 / 14)$ of human TSHR-immunized of the Z-score of the individual animals: control $ß$-Gal mice all showed a score $\leq 0$, as well as 2 of the TSHR-immunized mice (14\%); 8 of the TSHR-immunized mice showed a moderate score of $0-1(57 \%)$, while $4(29 \%)$ were scored $\geq 1$ (severe disease). The objective analysis showed no diseased $B-G a l$ control thyroids. 


\section{Discussion}

We describe a detailed analysis of the pathology of the thyroid gland in a mouse model of experimental GD [8, 9]. The disease was characterized by all features of GD such as increased serum levels of total $\mathrm{T}_{4}$ and the production of antibodies to TSHR. Moreover, the model was also accompanied by GO. The thyroid glands of the animals undergoing experimental GD were enlarged with typical signs of hyperthyroidism. We utilized the powerful technique of morphometric analysis using Cell Profiler to analyze in detail the histopathology of the hyperthyroidism in the induced animal model. To our knowledge, such detailed analysis of thyroid gland histopathology and morphometric analysis of the gland in an induced animal model of GD has not been reported previously.

We show that the thyroid glands in animals undergoing experimental GD closely reproduce the early changes of human thyroid pathology present in human GD patients $[18,19]$. The thyroid glands of GD patients are diffusely involved by follicular expansion and fundamentally no stromal changes. The thyroid glands in the mouse model reveal a noninflammatory diffuse follicular cell hyperplasia and hypertrophy that was supported by a significant increase of the follicular area of enlarged thyroid glands with increased follicle/stroma ratio. In general terms, hypertrophy refers to an increase in the size of cells, increasing the size of the organ, while hyperplasia is an increase in the number of cells in an organ or tissue, usually resulting in an increased mass of the organ or tissue. Although hyperplasia and hypertrophy are distinct processes, they frequently occur together, and they may be triggered by the same external stimulus such as stimulation by hormones and growth factors. The total glandular area was significantly increased in mouse GD glands, as well as the MFA. A previous study has revealed that primarily colloid-rich goiters may form in the presence of continuously higher than normal thyrotropin levels without a previous stage of follicular hyperplasia [20]. Our morphometric analysis highlights this aspect and proves that the accumulation of colloid is compatible with intense TSHR stimulation.

Thyroid follicular cells are capable of cell division and respond to stress or external signals (including TSHR antibodies) by undergoing hyperplasia and hypertrophy. GD follicular hyperplasia is the result of TSHR-driven proliferation of mature follicular cells by the autoantibodies without any histological evidence of tissue damage. This element represents an essential difference with Hashimoto thyroiditis (the other end of autoimmune

Follicular Hypertrophy/Hyperplasia of GD: Morphometric Evaluation thyroid disease). The hypertrophic component of this reaction pattern supports a morphofunctional activation of the follicular cells targeted by TSHR antibodies in the model. Our finding of an increase in follicle/stroma ratio in GD glands emphasizes a pathology dominated by follicular epithelial expansion with a minimal interstitial response. This approach also identifies the colloid storage hyperfunction status. In the absence of tissue injury and inflammation, the integrity of the extracellular matrix is a central factor to explain the diffuse hyperplastic reaction observed in GD. If tissue injury is severe or chronic and results in damage to both parenchymal cells and the stromal framework of the tissue, healing cannot be accomplished by regeneration. Under these conditions, the primary healing process is repaired by deposition of collagen and other extracellular matrix components, causing a variable degree of fibrosis and, sometimes, neoplastic transformation [21]. The morphometric evaluation of area/volume is the most sensitive quantitative method to assess the expansion of follicular cells. All these histological findings can be observed in Hashimoto thyroiditis and were absent in our GD model. Tissues with low proliferation reveal a highly variable response to external stimuli and their expansion is dominated by blocking apoptosis $[21,22]$. The quantitation of these aspects results in highly overlapping groups without statistical differences. The thyroid follicular epithelium has a low cellular turnover, even after stimulation by goitrogenic agents $[23,24]$. This finding assesses follicular cell proliferation as an unreliable variable to determine the follicular expansion due to the high standard deviation associated with topographically heterogeneous events. For this reason, the best option for this analysis is the determination of the follicular area related to the hypertrophic response, in concordance with the evidence that the accumulation of colloid is also an expression of intense TSHR stimulation [20].

Heterogeneity of GD thyroid in human pathology is well known and comprises a mixed initial hyperplastic reaction along with changes induced by the presurgical therapeutic intervention (i.e., Lugol) or antithyroid drug therapy. An increased proportion of active-like follicles, enlarged follicular cells, and the presence of papillae are all expressions of follicular expansion - by no means requirements for a morphological definition. The pathological response in GD thyroid glands is a purely cellular hyperplastic and hypertrophic reaction with no interstitial inflammation. It should be emphasized that the morphometric analysis correlated with the morphological evaluation but it was revealed to be more sensitive in 
identifying minor abnormalities using the follicle/stroma ratio as morphometric screening. The level of inflammatory reaction is quite variable in GD, most of the time only as a focal and nondestructive inflammation at a mild level. In human samples, a concomitant lymphocytic infiltrate can be observed interspersed between follicles or in the interstitial tissue. This lymphocytic infiltrate is predominantly a cytotoxic suppressor between the epithelial cells and mainly helper-inducer $\mathrm{T}$ cells in the interstitial tissue $[1,10]$. In both cases, the morphometric evaluation identifies this issue by a relative decrease of the follicle/ stroma ratio that can be confirmed by histological evaluation. Human samples are essentially not significantly inflamed and the level claimed in other models, although higher than controls, is of a modest level (up to 2 cells/ follicle), mainly of helper phenotype [25]. Many cases of florid inflammatory response reflect mixed autoimmune thyroiditis with follicular tissue destruction. At the other end of the spectrum, GD thyroid glands examined in human pathology frequently show follicular architecture with infolding, enlarged follicular cells and, occasionally, mild multifocal interstitial inflammation (if any). The changes we are describing herein replicate the human model. The vascular congestion and increased vascularity described in human samples are related to a presurgical treatment of hyperthyroid patients in preparation for the excision, and a reaction to tissue ischemia due to locally compressive expansile nodules. These two aspects were not present in our cohort and explain the absence of significant vascular changes. The presence of nodularity with ischemic-vascular changes and significant inflammatory infiltrate with local antigen modulation (germinal centers) are responsible for signs of oxidative stress [26, 27]. These are not essential and constant elements of GD. Human samples are available from GD patients treated surgically, and we should not include as primary features those changes related to preoperative therapy like stromal congestion or nodular transformation $[28,29]$. The histological finding of an increase in the follicle/stroma ratio would support an increase in follicular volume that, without evidence of deficient cell function, correlates with hyperthyroidism. In this scenario, persistent stimulation without receptor desensitization provides a potential explanation for the sustained hyperthyroid status as reported in our earlier study on an induced model of GD in humanized nonobese diabetic mice [30].

In summary, the thyroid glands of experimentally induced GD in animals immunized by electroporation of human TSHR A-subunit plasmid reproduce the human pathology counterpart. These thyroid glands reveal a noninflammatory diffuse follicular cell hyperplasia/hypertrophy that is supported by a significant increase of the follicular area of enlarged thyroid glands with an increased follicle/stroma ratio. The hypertrophic component of this reaction pattern supports a morphofunctional activation of the follicular cells targeted by TSHR antibodies. The lack of tissue damage and inflammation explains the absence of fibrosis and reparative processes. Overall, this mouse model is a faithful model of early changes in GD.

\section{Acknowledgments}

We thank Christoph Jesenek (Molecular Ophthalmology, University of Duisburg-Essen) for technical assistance. This project was funded by Interne Forschungsförderung Essen (IFORES).

\section{Statement of Ethics}

The protocol used in the study was approved by the Hospital Research Board and Ethics Committees and complied with their requirements.

\section{Disclosure Statement}

The authors of this paper have no competing financial interests.

\section{Author Contributions}

A.S., S.D.-C., and J.P.B. conceived and designed the study. A.S., A.B., and M.H. performed the experiments and statistical analysis. A.S., S.D.-C., J.P.B., A.E., and U.B.-P. analyzed and interpreted the data. S.D.-C., J.P.B., A.S., and A.B. drafted the manuscript. A.S., S.D.-C., J.P.B., A.E., U.B.-P., and S.L. revised the manuscript critically for important intellectual content. All authors read and approved the final paper.

References

\footnotetext{
1 Burman KD, Baker JR Jr: Immune mechanisms in Graves' disease. Endocr Rev 1985;6: 183-232.

2 Johnson JR: Adenomatous goiters with and without hyperthyroidism; some aspects of the relationship of the microscopic appearance to hyperthyroidism. Arch Surg 1949;59:10881099.

-3 Schicha H, Emrich D, Schreivogel I: Hyperthyroidism due to Graves' disease and due to autonomous goiter. J Endocrinol Invest 1985; 8:399-407.
}

$8 \quad$ Eur Thyroid J

DOI: $10.1159 / 000488079$
Schlüter et al. 
-4 Spjut HJ, Warren WD, Ackerman LV: Clinical-pathologic study of 76 cases of recurrent Graves' disease, toxic (non-exophthalmic) goiter, and nontoxic goiter; does a relation exist between thyroid hyperplasia and struma lymphomatosa? Am J Clin Pathol 1957;27: 367-392.

-5 Banga JP, Moshkelgosha S, BerchnerPfannschmidt $U$, Eckstein A: Modeling Graves' orbitopathy in experimental Graves' disease. Horm Metab Res 2015;47:797-803.

-6 Wiesweg B, Johnson KT, Eckstein AK, Berchner-Pfannschmidt $\mathrm{U}$ : Current insights into animal models of Graves' disease and orbitopathy. Horm Metab Res 2013;45:549-555.

-7 Braley-Mullen H, Yu S: NOD.H-2h4 mice: an important and underutilized animal model of autoimmune thyroiditis and Sjogren's syndrome. Adv Immunol 2015;126:1-43.

8 Berchner-Pfannschmidt U, Moshkelgosha S, Diaz-Cano S, Edelmann B, Gortz GE, Horstmann M, Noble A, Hansen W, Eckstein A, Banga JP: Comparative assessment of female mouse model of Graves' orbitopathy under different environments, accompanied by proinflammatory cytokine and T-cell responses to thyrotropin hormone receptor antigen. Endocrinology 2016;157:1673-1682.

-9 Moshkelgosha S, So PW, Deasy N, Diaz-Cano S, Banga JP: Cutting edge: retrobulbar inflammation, adipogenesis, and acute orbital congestion in a preclinical female mouse model of Graves' orbitopathy induced by thyrotropin receptor plasmid-in vivo electroporation. Endocrinology 2013;154:3008-3015.

10 Watson PF, Pickerill AP, Davies R, Weetman AP: Analysis of cytokine gene expression in Graves' disease and multinodular goiter. J Clin Endocrinol Metab 1994;79:355-360.

-11 Rapoport B, Chazenbalk GD, Jaume JC, McLachlan SM: The thyrotropin (TSH) receptor: interaction with TSH and autoantibodies. Endocr Rev 1998;19:673-716.

12 McLachlan SM, Aliesky HA, Pichurin PN, Chen CR, Williams RW, Rapoport B: Shared and unique susceptibility genes in a mouse model of Graves' disease determined in $\mathrm{BXH}$ and CXB recombinant inbred mice. Endocrinology 2008;149:2001-2009.

13 McLachlan SM, Nagayama Y, Pichurin PN, Mizutori Y, Chen CR, Misharin A, Aliesky HA, Rapoport B: The link between Graves' disease and Hashimoto's thyroiditis: a role for regulatory T cells. Endocrinology 2007;148: 5724-5733.

14 Ajjan RA, Weetman AP: The pathogenesis of Hashimoto's thyroiditis: further developments in our understanding. Horm Metab Res 2015;47:702-710.

15 Weetman AP: The immunopathogenesis of chronic autoimmune thyroiditis one century after Hashimoto. Eur Thyroid J 2013;1:243250.

16 Chen CR, Pichurin P, Nagayama Y, Latrofa F, Rapoport B, McLachlan SM: The thyrotropin receptor autoantigen in Graves disease is the culprit as well as the victim. J Clin Invest 2003; 111:1897-1904.

17 Schluter A, Horstmann M, Diaz-Cano S, Plohn S, Stahr K, Mattheis S, Oeverhaus M, Lang S, Flogel U, Berchner-Pfannschmidt U, et al: Genetic immunization with mouse thyrotrophin hormone receptor plasmid breaks self-tolerance for a murine model of autoimmune thyroid disease and Graves' orbitopathy. Clin Exp Immunol 2018;191:255-267.

18 LiVolsi VA: The pathology of autoimmune thyroid disease: a review. Thyroid 1994;4: 333-339.

19 Livolsi VA, Merino MJ: Histopathologic differential diagnosis of the thyroid. Pathol Annu 1981;16:357-406.

20 Gerber H, Studer H, Conti A, Engler H, Kohler H, Haeberli A: Reaccumulation of thyroglobulin and colloid in rat and mouse thyroid follicles during intense thyrotropin stimulation. A clue to the pathogenesis of colloid goiters. J Clin Invest 1981;68:1338-1347.

21 Arif S, Blanes A, Diaz-Cano SJ: Hashimoto's thyroiditis shares features with early papillary thyroid carcinoma. Histopathology 2002;41: 357-362.

22 Diaz-Cano SJ: Kinetic topographical heterogeneity in follicular thyroid neoplasms and growth patterns. Histopathology 2007;51: 416-418.

23 Dumont JE, Maenhaut C, Lamy F: Control of thyroid cell proliferation and goitrogenesis. Trends Endocrinol Metab 1992;3:12-17.

24 Williams DW, Wynford-Thomas D, Williams ED: Control of human thyroid follicular cell proliferation in suspension and monolayer culture. Mol Cell Endocrinol 1987;51:3340.

25 Costagliola S, Many MC, Denef JF, Pohlenz J, Refetoff S, Vassart G: Genetic immunization of outbred mice with thyrotropin receptor cDNA provides a model of Graves' disease. J Clin Invest 2000;105:803-811.

26 Erem C, Suleyman AK, Civan N, Mentese A, Nuhoglu I, Uzun A, Ersoz HO, Deger O: Ischemia-modified albumin and malondialdehyde levels in patients with overt and subclinical hyperthyroidism: effects of treatment on oxidative stress. Endocr J 2015;62:493-501.

27 Marique L, Van Regemorter V, Gerard AC, Craps J, Senou M, Marbaix E, Rahier J, Daumerie C, Mourad M, Lengele B, et al: The expression of dual oxidase, thyroid peroxidase, and caveolin-1 differs according to the type of immune response (TH1/TH2) involved in thyroid autoimmune disorders. J Clin Endocrinol Metab 2014;99:1722-1732.

28 Carnell NE, Valente WA: Thyroid nodules in Graves' disease: classification, characterization, and response to treatment. Thyroid 1998;8:647-652.

29 Chang DC, Wheeler MH, Woodcock JP, Curley I, Lazarus JR, Fung H, John R, Hall R, McGregor AM: The effect of preoperative Lugol's iodine on thyroid blood flow in patients with Graves' hyperthyroidism. Surgery 1987; 102:1055-1061.

30 Flynn JC, Gilbert JA, Meroueh C, Snower DP, David CS, Kong YC, Banga JP: Chronic exposure in vivo to thyrotropin receptor stimulating monoclonal antibodies sustains high thyroxine levels and thyroid hyperplasia in thyroid autoimmunity-prone HLA-DRB $1 * 0301$ transgenic mice. Immunology 2007;122:261267. 